\title{
EDITORIAL
}

\section{Launch of the NSFC Excellent Young Scholars Forum}

\author{
Zhi-Hua ZHOU \\ Executive Editor-in-Chief \\ National Key Laboratory for Novel Software Technology, Department of Computer Science \& Technology, \\ Nanjing University, Nanjing 210023, China
}

(c) Higher Education Press and Springer-Verlag Berlin Heidelberg 2015

In 2012, the National Natural Science Foundation of China (NSFC) launched the Excellent Young Scholars (EYS) Program. As its name suggests, this program aims to recognize and support excellent young scholars in the fields of science and engineering. This program is similar to the NSF Career Award in the United States, and the competition is very tough: only a very limited number of applicants can get through. Each awardee will receive a fund of one million CNY for a three-year term. With such generous support, awardees are expected to do more excellent research and grow up quickly as distinguished young scholars.

There is no doubt that the awardees of the NSFC-EYS Program in computer science fields are excellent representatives of the young generation of Chinese computer scientists. To introduce them to the broader community, Frontiers of Computer Science launches this new forum. The leading authors (either the first authors or the communication authors) of the articles in this forum are NSFC-EYS awardees. The articles either review the awardees' research fields from their own viewpoints, or summarize their own lines of research, or present their recent research advances.

For the first time of the NSFC Excellent Young Scholars Forum, two articles are published in this issue. The first article, "Large-scale virtual machines provisioning in clouds: challenges and approaches" by an awardee of the NSFC-EYS Program in 2012, Dr. Dongsheng Li (and his student and colleague), reviews existing provisioning schemes for virtual machines. This article classifies these schemes into three

Received November 1, 2015

E-mail: zhouzh@nju.edu.cn categories and discusses features and research status of each category. Two recent solutions are also given by the authors.

The second article, "Scene text detection and recognition: recent advances and future trends" by another awardee of the NSFC-EYS Program in 2012, Dr. Xiang Bai (and his students), provides a survey on scene text detection and recognition. This article not only introduces state-of-the-art techniques, but also predicts potential future research directions. It also offers comprehensive links to research resources, including datasets, source codes and online demos.

We sincerely hope that the NSFC Excellent Young Scholars Forum will be able to serve as a window of exhibiting researches and visions of the excellent young generation computer scientists of China, and hope that this forum will become an attractive feature of Frontiers of Computer Science. 